山्山FANÇAISE

$>\mathrm{DE}$

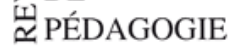

\section{Revue française de pédagogie}

Recherches en éducation

168 | juillet-septembre 2009

Enseignement et apprentissages, entre psychologie et didactiques

\title{
LES COMPAGNONS. L'Université nouvelle [édition critique par Bruno Garnier]| GARNIER Bruno. Les combattants de l'école unique
}

Lyon : INRP, 2008. - 385 p.| Lyon : INRP, 2008. - 351 p.

Pierre Kahn

\section{(c) OpenEdition}

Édition électronique

URL : http://journals.openedition.org/rfp/1847

DOI : $10.4000 / \mathrm{rfp} .1847$

ISSN : 2105-2913

Éditeur

ENS Éditions

Édition imprimée

Date de publication : 1 juillet 2009

Pagination : 145-147

ISBN : 978-2-7342-1172-3

ISSN : 0556-7807

Référence électronique

Pierre Kahn, « LES COMPAGnons. L'Université nouvelle [édition critique par Bruno Garnier]| garnier Bruno. Les combattants de l'école unique », Revue française de pédagogie [En ligne], 168 | juillet-septembre 2009, mis en ligne le 07 octobre 2010, consulté le 25 septembre 2020. URL : http://journals.openedition.org/ rfp/1847; DOI : https://doi.org/10.4000/rfp.1847

Ce document a été généré automatiquement le 25 septembre 2020

(c) tous droits réservés 


\title{
LES COMPAGNONS. L'Université nouvelle
} [édition critique par Bruno Garnier]| GARNIER Bruno. Les combattants de l'école unique

Lyon : INRP, 2008. - 385 p.| Lyon : INRP, 2008. - 351 p.

\author{
Pierre Kahn
}

\section{RÉFÉRENCE}

LES COMPAGNONS. L'Université nouvelle [édition critique par Bruno Garnier]. Lyon : INRP, 2008. $-385 \mathrm{p}$.

GARNIER Bruno. Les combattants de l'école unique. Lyon : INRP, 2008. - 351 p.

1 Mettre à disposition du public et des chercheurs, avec l'appareil critique nécessaire, des écrits plus cités que lus (premier ouvrage) et les accompagner d'un commentaire à la fois historique et analytique permettant d'en comprendre le sens et les enjeux complexes et évolutifs (deuxième ouvrage) : tel est le résultat du travail, ô combien salutaire, de Bruno Garnier sur les Compagnons de l'Université nouvelle. Ce qu'on savait d'eux en général, avant ce livre, se résumait à peu de choses : ils étaient des anciens combattants partisans déclarés de l'école unique et de la démocratisation de l'enseignement dont le plan Langevin-Wallon, à la Libération, a reçu l'héritage. Mais que signifie pour les Compagnons "école unique» et "démocratisation»? Ces universitaires et ces enseignants issus des tranchées, qui étaient-ils au juste ? Comment enfin ce groupe, d'abord confidentiel (sept personnes), s'est-il étoffé jusqu'à atteindre un millier de personnes au début des années 1920, et comment a-t-il vu ses conceptions évoluer? C'est tout cela qu'on savait beaucoup moins et que, grâce au travail à la fois synthétique et scrupuleux de Bruno Garnier, on est davantage inexcusable aujourd'hui de ne pas savoir. Je rendrai compte ici spécialement du livre de Bruno Garnier, en ne 
renvoyant à l'édition critique des textes de l'Université nouvelle parus chez Fischbacher de 1918 à 1924 que comme à un corpus de référence.

2 L'auteur, avec un souci de contextualisation véritablement remarquable, s'emploie d'abord (chapitre I) à mettre en évidence les thèses du moment de ce qu'il appelle la «fabrication» du groupe et de sa doctrine. La démocratisation de l'enseignement ne consiste pas à permettre à chacun de développer autant qu'il le peut, et dans des conditions égales pour tous, ses potentialités, mais à adopter le point de vue de l'intérêt général : la patrie, surtout au lendemain de la guerre, ne doit pas passer à côté d'un talent ou d'une intelligence utile. L'exigence d'unité prime donc celle d'égalité (p. 91). Pour les Compagnons du début des années 1920, l'école unique est au service de l'unité de la nation et son grand ennemi est la lutte des classes. L'école doit être le foyer du renouveau du civisme républicain (p. 91 sqq.). Le programme de l'Université nouvelle n'est pas socialiste mais s'inscrit bien plutôt dans la continuité républicaine. Il s'agit d'établir dans les faits le projet politique et moral de l'école de Jules Ferry (p. 25).

3 La démocratisation souhaitée n'implique aucune rupture avec les finalités traditionnelles de l'enseignement secondaire: la sélection des élites. Elle consiste seulement à substituer une sélection par le mérite et les capacités à une sélection par l'argent (voir par exemple p.69), même si Bruno Garnier note, p.79, que les compagnons sont favorables à une diversification des élites. L'école unique doit donc permettre de former, sur une base méritocratique, d'une part des producteurs - ceux qui en son sein n'auront pas les capacités requises pour intégrer l'enseignement secondaire -; d'autre part la future élite dirigeante (p. 77 sqq.). Et l'enseignement secondaire auxquels seuls ceux-ci sont destinés ne peut être que classique, c'est-à-dire dominé par l'étude des langues anciennes. Les Compagnons, au sein desquels l'enseignement primaire est très peu représenté (p.59), récusent l'idée d'« humanités scientifiques » sur laquelle Louis Liard avait pourtant, en 1902, construit la réforme de l'enseignement secondaire masculin (voir p. 67-68, p. 80 sqq.).

4 L'école des Compagnons est certes unique en ce qu'elle vise à abolir les cloisons sociales qui séparaient alors de façon étanche les ordres d'enseignement (primaire pour le peuple, secondaire pour les notables) et en ce qu'elle oblige tous les enfants à passer par un enseignement primaire unifié (Bruno Garnier ne manque pas de remarquer, p. 71 et suivantes, que les Compagnons sont pour la suppression de l'enseignement primaire supérieur). Mais elle ne l'est pas en ce qu'elle voudrait une scolarité secondaire prolongée pour tous. Il y a de ce point de vue une différence importante entre le programme des Compagnons et les propositions de la commission LangevinWallon, sur laquelle l'auteur aurait pu davantage insister. Je crois que s'il ne l'a pas fait, c'est parce que son livre a tendance à majorer l'influence des Compagnons sur le plan Langevin-Wallon et la continuité des vues existant des uns à l'autre. Je reviendrai plus loin sur ce point.

5 Le principe de l'école unique, aussi fortement affirmé soit-il, n'empêche pas les Compagnons d'être favorables à une localisation laissant les enfants dans leur environnement scolaire et social propre: bourgeois au lycée, paysans à l'école du village, peuple des villes à l'école du quartier (p. 24). De sorte que si l'école est unique par sa structure, elle ne permet pas pour autant le mélange des classes dont les Compagnons disaient pourtant qu'il devait être le fait de l'école en temps de paix comme il avait été celui des tranchées en temps de guerre. Il y a là comme un repentir 
de l'idéal démocratique que les Compagnons, note Bruno Garnier, semblent avoir hésité à rêver jusqu'au bout (voir par exemple p. 91).

6 L'école unique des Compagnons trouve son modèle dans l'Einheitsschule allemande (p. 61), variante d'une « xénophilophobie » fort répandue après la défaite de 1870 : il ne faut pas laisser à l'ennemi l'avantage de ses forces, que les Compagnons connaissaient d'ailleurs bien, puisque quatre d'entre les sept membres fondateurs étaient des germanistes (p.36). L'organisation de l'enseignement doit selon les Compagnons reposer sur la constitution d'une corporation enseignante intégrant le privé et administrant l'Université de façon décentralisée (p. 91 sqq.).

$7 \mathrm{Au}$ total, l'analyse est assez démystificatrice: conservation de l'enseignement secondaire classique, de ses finalités comme de l'«aristocratisme» (p. 83) de sa culture; limitation de la démocratisation à la méritocratie; unité structurelle de l'école, mais sans réel brassage social des enfants ; revendication de l'héritage moral et politique du projet scolaire de Jules Ferry, dont les Compagnons ne critiquent que l'absence de réalisation véritable. En effet les « combattants de l'école unique » ne sont pas, tant s'en faut, des militants de l'émancipation de la classe ouvrière ; il y a même loin de leur conception de la démocratisation à la thématique de l'égalité des chances qui sert à la définir aujourd'hui (p. 63 sqq.). Bruno Garnier l'écrit nettement: «la réparation et l'atténuation des inégalités d'origine sociale ne faisaient tout simplement pas partie de leurs intentions » (p. 71).

8 La « réception » du programme de l'Université nouvelle (chapitre II) rend compte des infléchissements ultérieurs de la doctrine. Malgré l'accueil favorable du catholicisme social (p. 108 sqq.), qui fut l'une des sources intellectuelles du mouvement (p. 100), la " répulsion des catholiques ", héritiers des "anti-Lumières " de Maistre et de Bonald (p. 113), et d'autre part « l'adhésion critique de la gauche» (p. 127), où ils vont trouver de «nouvelles alliances» (p.157), vont conduire les Compagnons vers la gauche du spectre politique. Une gauche certes réformiste : le refus de la lutte des classes rend leurs positions définitivement incompatibles avec la gauche révolutionnaire (p.136 sqq.). Le rapprochement avec le syndicalisme réformiste (p.145) témoigne de cette politique que le chapitre III ( L'action ») décrit en détail : liens tissés avec les radicauxsocialistes (p.157), adhésion des grands partis de gauche et des associations démocratiques (Ligue des droits de l'homme, Ligue de l'enseignement, etc.) au principe de l'école unique (p. 166); dénonciation de la suppression de la section moderne des lycées conçue en 1923-1924 par Léon Bérard (p.167 sqq.); accompagnement (certes critique) de la réforme de l'école unique voulue par le Cartel des gauches (p. 213 sqq.) ; sensibilité de plus en plus marquée, sous l'influence du rapprochement avec la CGT, à la problématique d'une école soucieuse autant de la masse que des élites (p. 274), et critique de plus en plus virulente du conservatisme académique des professeurs de l'enseignement secondaire (p. 276-278), etc. Corrélativement, la doctrine se transforme sur des points décisifs : révision de la position originelle sur les humanités scientifiques (liée au refus de la réforme Bérard), reconnaissance de l'enseignement primaire supérieur et d'une troisième voie possible entre humanités secondaires et formation professionnelle (p. 203), substitution de la problématique de l'orientation à celle de la sélection (p. 225 sqq.), etc.

9 La personne et l'action de Paul Langevin, successeur d'Henri Laugier à la tête de l'association à partir de 1930, illustrent cette évolution politique des Compagnons. Et Langevin n'est évidement pas pour rien, Bruno Garnier y insiste avec justesse (p. 289 
sqq.), dans le rapprochement opéré avec le mouvement de l'Éducation nouvelle et avec les pédagogies actives auxquelles les Compagnons de la fin de la Grande Guerre étaient restés finalement assez peu sensibles. On comprend alors le lien affirmé dès le début de l'ouvrage entre les Compagnons et la commission Langevin instituée à la Libération. Peut-être cependant, je le disais au début de cette notice, l'auteur surestime-t-il ce lien. La continuité existante entre le projet des Compagnons et les perspectives de la commission de la réforme de l'enseignement mise en place en 1944 est certes réelle, et est comme incarnée par la personne même de celui qui fut le président de cette commission, mais les différences n'en sont pas moins importantes, notamment sur la conception même de la démocratisation. Pour les Compagnons, démocratiser signifie diversifier les élites - ce que Langevin lui-même rappelle encore lorsqu'il est à la tête de l'association (p. 295). Mais pour la commission à laquelle le même Langevin a donné son nom, démocratiser signifie autre chose : l'élévation de tous aux diverses dimensions de la culture universelle et l'épanouissement des aptitudes individuelles différentes mais égales, quelle que soit la position sociale, c'est-à-dire en somme une conception non méritocratique de la justice scolaire - ce qui a conduit par exemple la commission Langevin à se prononcer contre l'examen d'entrée en $6^{\mathrm{e}}$ auquel les Compagnons ont été quant à eux favorables (p. 263 sqq.).

10 Entre la démocratisation de la sélection défendue dès 1918 par les Compagnons et la « démocratisation de la réussite » version années 1960-1970, le plan Langevin-Wallon constitue un autre modèle de justice scolaire. Il manque peut-être au livre de Bruno Garnier, qui a l'ambition légitime d'être une contribution à une généalogie de la démocratisation de l'enseignement en France - et qui est globalement, c'est certain, à la hauteur d'une telle ambition - de saisir toute la complexité des liens qui unissent et séparent à la fois ces différentes conceptions de la démocratisation. Il lui manque aussi de ne pas poser la question du rapport des Compagnons avec le pacifisme de l'entredeux-guerres et de ne jamais évoquer le problème de l'enseignement secondaire féminin. Il est vrai d'une part que le modèle " militaire » de civisme républicain prôné par l'Université nouvelle au sortir de la guerre ne convient guère aux jeunes filles; d'autre part que la préoccupation d'une sélection méritocratique des futures élites sociales conduit à ne pas trop se soucier de l'enseignement féminin. Mais cela aurait été justement une autre occasion d'interroger de façon critique la conception que les Compagnons se sont faits de la démocratisation et de l'école unique.

11 Ces remarques procèdent de l'envie que l'on a de continuer la discussion ouverte avec Bruno Garnier sur cette période cruciale de l'histoire de la démocratisation scolaire, dont il a permis de mieux connaître certains des acteurs et certaines des doctrines qui étaient jusqu'ici davantage des révérences que des références. Il faut donc saluer, avec le livre de Bruno Garnier et la mise à disposition des textes de l'Université nouvelle, une contribution historiographique importante et éclairante. 


\section{AUTEURS}

PIERRE KAHN

CERSE, université de Caen Basse-Normandie 